\title{
2015 Morgan Prize
}

LEVENT ALPOGE was awarded the Frank and Brennie Morgan Prize for Outstanding Research in Mathematics by an Undergraduate Student at the 121st Annual Meeting of the AMS in San Antonio, Texas, in January 2015.

\section{Citation}

Levent Alpoge is the recipient of the 2015 AMSMAA-SIAM Frank and Brennie Morgan Prize for Outstanding Research in Mathematics by an Undergraduate Student for several contributions in the fields of number theory, probability, and combinatorics. As an undergraduate, his senior thesis on the average number of integral points in elliptic curves received the Captain Jonathan Fay Prize for the best senior thesis written by a Harvard undergraduate. He has authored or coauthored seven papers, where he has established a substantial record of proposing innovative solutions for difficult problems.

Alpoge participated in two Research Experiences for Undergraduates (REUs), one at Williams College in 2012 and the other at the University of Minnesota at Duluth in 2013. Several of his publications resulted from projects that were first initiated in these REUs.

In addition to the Captain Jonathan Fay Prize at Harvard, Alpoge has received many other prizes, including the Sophia Freund Prize, the Thomas T. Hoopes Prize, the David Mumford Prize, and the Churchill Scholar Award.

\section{Biographical Sketch}

Levent Alpoge was born and raised in Dix Hills, New York. He is a graduate of the Long Island School for the Gifted, Half Hollow Hills High School West, and Harvard University, where he obtained an A.B. in mathematics and an AM in physics through advanced standing. He is currently at Cambridge University undertaking Part III of the Mathematical Tripos as a Churchill Scholar, and he will enter Princeton's PhD program in the fall. His mathematical focus is number theory, which he first discovered at the Ross Program at the Ohio State University.

While at Harvard, Alpoge took many courses in mathematics, physics, and computer science. He spent the summer of 2011 at the PRISE program at Harvard on a Herchel Smith fellowship, studying algebraic number theory under Benedict Gross. He spent his next two summers at the Williams SMALL and Duluth REUs, working on problems in analytic number theory and combinatorics under Steven Miller and Joe Gallian, respectively. During this time, he also worked as a course assistant for mathematics courses at Harvard,

DOI: http://dx.doi.org/10.1090/noti1229 obtaining certificates for distinction in teaching; he gave talks at various conferences as well as Harvard's own Math Table; and he taught eight- to ten-year-olds at Bob and Ellen Kaplan's Math Circle.

His nonmathematical pursuits include playing soccer, distance running, and reading histories and biographies.

Response from Levent Alpoge It is an incredible honor to receive the 2015 AMS-MAA-SIAM Frank and Brennie Morgan Prize

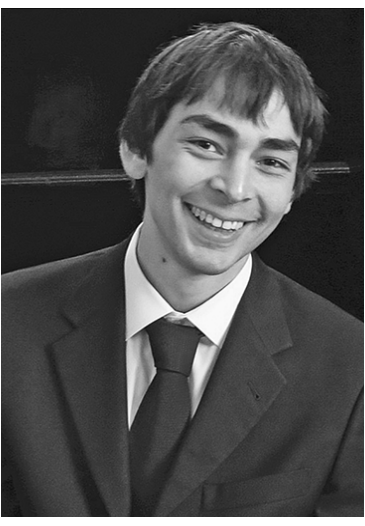

Levent Alpoge for Outstanding Research in Mathematics by an Undergraduate Student. I would first like to thank Mrs. Morgan and the AMS, MAA, and SIAM for this award and for encouraging undergraduate research in mathematics. I would also like to thank Steven Miller and Joe Gallian both for advising me and for running such superb REUs at Williams College and the University of Minnesota-Duluth. I would next like to thank all my teachers at LISG, Hills West, and Harvard, particularly Mrs. Kapner at LISG, Mr. Blayne, Mr. Maroney, and Mrs. Notskas at Hills West, and Professors Elkies, Gaitsgory, and Gross at Harvard for their patient and unselfish teaching throughout my education. I would like to also thank Jacob Tsimerman, my senior thesis adviser, and Arul Shankar for both devising a wonderful problem for my senior thesis and patiently explaining and reexplaining the beautiful mathematics surrounding it to me. On the topic of my thesis, I would like to thank Professor Gallian (again!) for getting me to ask for a thesis problem in the first place, since I would have thought it out of the question were it not for his encouragement. I would of course like to thank my friends for making life so fun, and, most importantly, I would like to thank my family for their constant love and support.

Citation for Honorable Mention: Akhil Mathew Akhil Mathew is recognized with an Honorable Mention for the 2015 Morgan Prize for Outstanding Research in Mathematics by an Undergraduate Student. Mathew's areas of research are algebraic topology, algebraic geometry, and category theory. He has received special recognition for his senior thesis at Harvard University titled "The Galois group of homotopy theory", in which he has introduced several new ideas in descent theory and Galois theory for structuring ring spectra.

Mathew has been the recipient of several prizes while at Harvard, including the David Mumford Prize and the Thomas T. Hoopes Prize. He 


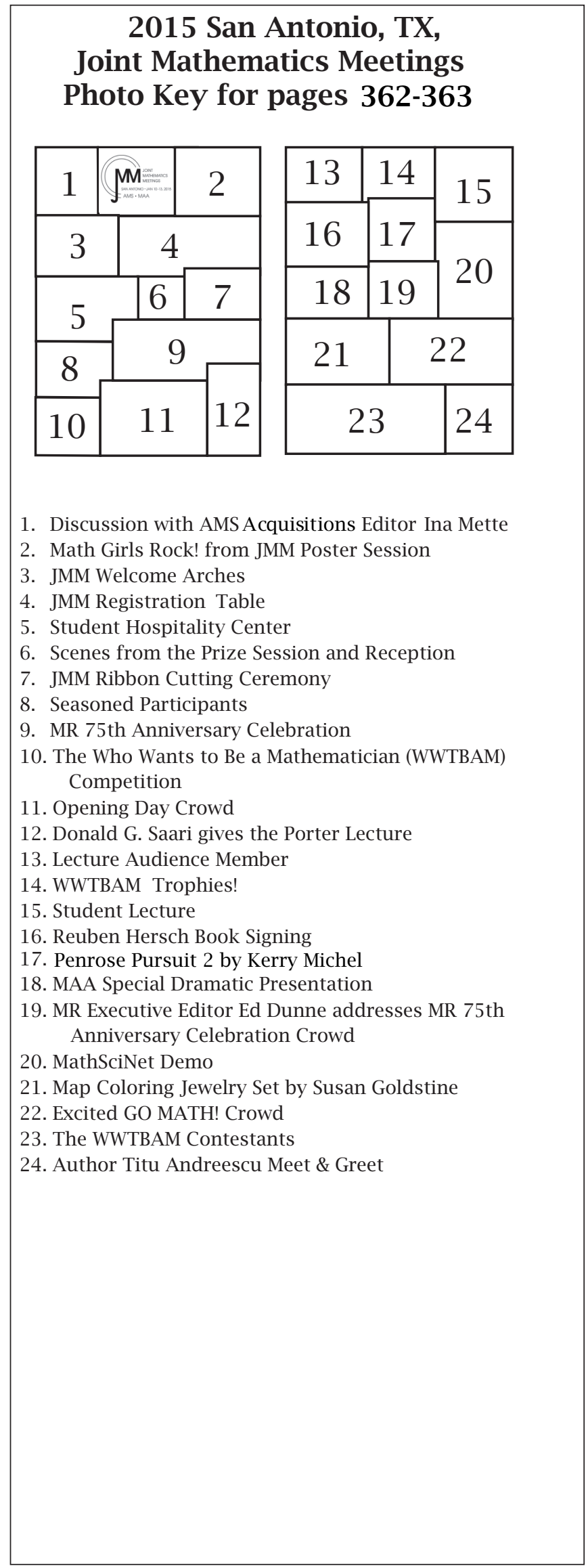

participated in two REUs, one in 2013 at Emory University and the other at Pennsylvania State University in 2010.

\section{Biographical Sketch}

Akhil Mathew was born in Chennai, India, but grew up in Madison, New Jersey. He became interested in mathematics as a child through popular books. As a high school student, he took university-level classes, and he began participating in mathematics research. He received third place in the Intel Science Talent Search in 2010 for this research. During college, he participated in REU programs at Pennsylvania State University and at Emory University, and he did summer research at Harvard through internal fellowships. In 2014 he earned a BA in pure mathematics from Harvard University. He is now a first-year graduate student at the University of California, Berkeley, supported by an NSF Graduate Fellowship. His primary mathematical interests are in algebraic topology, algebraic geometry, and higher category theory.

\section{Response from Akhil Mathew}

It is an honor and a privilege to receive Honorable Mention for the 2015 AMS-MAA-SIAM Frank and Brennie Morgan Prize for Outstanding Research in Mathematics by an Undergraduate Student. I would like to thank my many mentors and collaborators, and in particular Dennis Gaitsgory, Mike Hopkins, and Jacob Lurie for their help and support at Harvard.

\section{About the Prize}

The Morgan Prize is awarded annually for outstanding research in mathematics by an undergraduate student (or students having submitted joint work). Students in Canada, Mexico, or the United States or its possessions are eligible for consideration for the prize. Established in 1995, the prize was endowed by Mrs. Frank (Brennie) Morgan of Allentown, Pennsylvania, and carries the name of her late husband. The prize is given jointly by the AMS, the Mathematical Association of America (MAA), and the Society for Industrial and Applied Mathematics (SIAM) and carries a cash award of US\$1,200.

Recipients of the Morgan Prize are chosen by a joint AMS-MAA-SIAM selection committee. For the 2015 prize, the members of the selection committee were Bela Bajnok, Jacob Fox, Johnny Guzman, Steven J. Leon, Reza Malek-Madani, and Susan E. Martonosi.

Previous recipients of the Morgan Prize are Kannan Soundararajan (1995), Manjul Bhargava (1996), Jade Vinson (1997), Daniel Biss (1998), Sean McLaughlin (1999), Jacob Lurie (2000), Ciprian Manolescu (2001), Joshua Greene (2002), Melanie Wood (2003), Reid Barton (2005), Jacob Fox (2006), Daniel Kane (2007), Nathan Kaplan (2008), Aaron Pixton (2009), Scott Duke Kominers (2010), Maria Monks (2011), John Pardon (2012), Fan Wei (2013), and Eric Larson (2014). 